\title{
Study on Processing Technology of Rose Milk Beverage
}

\author{
Fan Dan-min ${ }^{1,2}$, Yang Zhi-long ${ }^{1,2}$ \\ ${ }^{1}$ Institute of Technolog, Lijiang Teachers College, Lijiang, China \\ ${ }^{2}$ Research Center for Plateau Characteristic Agriculture in Northwest Yunnan Province, Lijiang, China
}

\begin{abstract}
The main raw materials of rose milk beverage are Yunnan red roses and milk. By analyzing the proportions of rose juice to milk, sucrose, citric acid and sodium alginate, and by taking flavor, taste, color and tissue state as the evaluation parameters, we conducted single-factor experiments and orthogonal experiments, and identified the optimum formula of rose milk beverage: $8 \%$ rose juice, $6 \%$ milk, $10 \%$ sucrose, $0.1 \%$ citric acid, and $0.15 \%$ compound stabilizer (sodium carboxymethyl cellulose, sodium alginate in the proportion of 1:1). Unique flavor of rose milk beverage with rich nutrition can be prepared by using the optimum formula we obtained.
\end{abstract}

\section{Introduction}

A rose is a woody perennial flowering plant of the genus Rosa in angiosperns, dicotyledonous plants, in the family Rosaceae. There are over 150 species around the world and 100 species in China.

Rose belongs to the genus Rosaceae in angiosperms, dicotyledonous plants, Rosaceae. There are about 150 species in the world and 100 species in China. Roses are prickle shrubs and its leaves are pinnate, wrinkled, with small prickles on the stem. The flowering period of the rose is from May to September each year, with solitary or 3-6 sepals ${ }^{[1]}$. The flowers of most species have one petal or five petals, with the colour in rose red. Roses are best known as ornamental plants grown for their flowers in the gardern. They have also been used for commercial perfumery and commercial cut lower crops for food processing. According to relevant research, roses taste subtle, sweet and slightly bitter, warm-natured, and emit fragrance ${ }^{[2]}$. Roses are not only rich in vitamins, tannic acid, natural pigments, but also are full of eight kinds of amino acids and many micoelements needed by the human body. Therefore, proper consumption of roses can replenish a variety of nutrients needed by the human body. The edible and medicinal value of roses is mainly maintaining beauty, nourishing the skin, promoting blood circulation to restore menstrual flow, nourishing the liver and alleviating anxiety. The main products of roses are rose flower cake, rose flower yoghurt, rose fruit cake, rose sugar, rose tea and rose compound beverage and so on.

\section{Test materials and methods}

\subsection{Materials (raw materials and ingredients)}

Yunnan red rose, milk, sugar, citric acid, carboxymethylcellulose sodium, sodium alginate, distilled water.

\subsection{Instrumenttation}

Electronic balance, juicer, gauze, glass bottle, stainless steel pot

\subsection{Process Flow}

$$
\text { milk } \rightarrow \text { pretreatment }
$$

$\downarrow$

rose $\rightarrow$ pretreatment $\rightarrow$ Juice percolation $\rightarrow$ collect filtrate $\rightarrow$ blending $\rightarrow$ homogenate $\rightarrow$ filling $\rightarrow$ sterilization $\rightarrow$ finish ed product

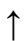

white sugar, citric acid, compound stabilizer

\subsection{Key Operation Points}

\subsubsection{Rose Pretreatment}

The first step is to select fresh edible Yunnan red roses, pick up their petals, rinse the petals in clean water, dry them and wait for use.

\subsubsection{Juice extraction and filtration}

The second step is to put dried rose petals in the juicer, filter them with clean gauze, and collect the filtrate.

\subsubsection{Milk pretreatment}

The third step is to choose fresh, disinfectant milk in milky white or slightly yellowish, with the essential aroma of fresh milk, which is presented as uniform fluid, without sediment, peculiar smell and viscous phenomenon. 


\subsubsection{Blending}

Blending is the key procedure in the processing of rose milk beverage, with strict requirements. It is necessary not only to accurately control the amount of raw materials and ingredients, but also to control the temperature and $\mathrm{pH}$ value to prevent protein denaturation and ensure the formation of uniform and stable beverage. The specific operation of blending is to take a certain amount of rose juice and milk with dosage cup, then weigh a certain amount of sugar, citric acid and stabilizer with electronic balance, mix the rose juice and milk evenly, and then add each ingredient in a certain amount to the mixture.

\subsubsection{Homogenization}

The fifth step is to pour the mixed rose milk into the homogenizer and wait for 5 minutes, in order to achieve homogenization and subtleness, and to avoid stratified precipitation.

\subsubsection{Filling and Exhausting}

The homogenized beverage was filled in a washed and sterilized glass bottle, which was placed vertically in a constant temperature water pot. The bottle cap was placed on the mouth of the bottle, without sealed. Let it exhaust in the constant temperature water pot at $60{ }^{\circ} \mathrm{C}$ for 30 minutes.

\subsubsection{Sterilization}

The seventh step is to screw the bottle cap after exhaust, and to put it in a constant temperature water pot at $95{ }^{\circ} \mathrm{C}$ for 30 minutes for sterilization.

\subsubsection{Cooling and finished product}

The sterilized beverage was cooled to below $40{ }^{\circ} \mathrm{C}$ to obtain the finished product.

\subsection{Sensory Evaluation}

The quality of the rose milk beverage is evaluated by four sensory indicators, that is, taste, color, aroma and structure state, with full score of 100 points. Each one of them scored 25 points. The scoring standards are shown in Table 1 .

Table 1 Sensory evaluation criteria for rose milk beverage

\begin{tabular}{lll}
\hline Sensory index & Standard for evaluation & Score \\
\hline \multirow{3}{*}{ Color } & Presenting rose red, clear & $21-30$ points \\
& Pink, not clear enough & $11-20$ points \\
& Darker shade & $1-10$ points \\
& The fragrance of the rose is prominent, no smell & $31-40$ points \\
Fragrance and taste & The fragrance of the rose is not prominent, with obvious odor. & $20-30$ points \\
& No fragrance, obvious odor & $1-19$ points \\
\multirow{5}{*}{ Appearance } & Clear and bright, no precipitation & $21-30$ points \\
& Slightly turbid, with a small amount of suspended matter & $11-20$ points \\
& The color is turbid, and the precipitation is excessive & $1-10$ points \\
\hline
\end{tabular}

\section{Results and Analysis}

\subsection{Single-factor test of beverage blending}

\subsubsection{Effect of rose juice volume on the quality of rose milk beverage}

The proportion of the rose juice has an effect on the taste, color, aroma and structure state of the rose milk beverage. Under the condition of a certain raw material, the proportion of rose juice is selected and blended by $4 \%, 6 \%$, $8 \%, 10 \%, 12 \%$ of the total amount. The effect of rose juice amount on the quality of rose milk beverage is shown in Table 2.
Table 2 Effect of rose juice volume on the quality of rose milk beverage

\begin{tabular}{ll}
\hline Dosage $(\%)$ & Sensory score/points \\
$4 \%$ & 16 \\
$6 \%$ & 18 \\
$8 \%$ & 23 \\
$10 \%$ & 21 \\
$12 \%$ & 20 \\
\hline
\end{tabular}

Table 2 shows that when the proportion of rose juice is between $2 \%$ and $6 \%$, the sensory evaluation score rises with the increase of rose juice. When the proportion of rose juice is $6 \%$, the sensory score is the highest. However, when the proportion is between $6 \%$ and $10 \%$, the score decreases gradually because of the darker color and stronger taste. Thus, the best proportion of rose juice is $6 \%$. 


\subsubsection{Effect of milk volume on the quality of rose milk beverage}

The volume of milk affects the taste and structure state of rose milk beverage. The more milk is added, the stronger milk smell accompanies. Meanwhile, rose milk beverage is not stable and easy to produce precipitation. The proportion of milk is selected at $2 \%, 4 \%, 6 \%, 8 \%, 10 \%$ of the total. The effect of the volume of milk on sensory evaluation is shown in Table 3 .

Table 3 Effect of milk volume on the quality of rose milk beverage

\begin{tabular}{ll}
\hline Dosage $(\%)$ & Sensory score/points \\
\hline $2 \%$ & 17 \\
$4 \%$ & 20 \\
$6 \%$ & 24 \\
$8 \%$ & 22 \\
$10 \%$ & 21 \\
\hline
\end{tabular}

Table 3 shows that when the proportion of milk is between $2 \%$ and $6 \%$ in the total, the sensory evaluation score rises with the increase of milk. When the proportion is $6 \%$, the sensory score is the highest. However, when the proportion is between $6 \%$ and $10 \%$, the score decreases gradually, owing to stronger smell of milk with some precipitation.

\subsubsection{Effect of white sugar volume on the quality of rose milk beverage}

Under the condition that $8 \%$ rose juice and $6 \%$ milk is added, we add sugar in different proportions of $6 \%, 8 \%$, $10 \%, 12 \%$, and $14 \%$ to prepare the rose milk beverage. The effect of white sugar volume on sensory evaluation scores is shown in Table 4.

Table 4 Effect of white sugar volume on the quality of rose milk beverage

\begin{tabular}{ll}
\hline Dosage $(\%)$ & Sensory score/points \\
$6 \%$ & 18 \\
$8 \%$ & 20 \\
$10 \%$ & 23 \\
$12 \%$ & 22 \\
$14 \%$ & 21 \\
\hline
\end{tabular}

Table 4 shows that when the proportion of white sugar is between $6 \%$ and $10 \%$, the sensory score rises with the increase of white sugar. When the proportion reaches $10 \%$, the sensory score is the highest. However, when the proportion is between $10 \%$ and $14 \%$, the score decreases gradually, because it's sickly sweet and cloying.

\subsubsection{Effect of citric acid volume on the quality of rose milk beverage}

Citric acid is widely used as a food acidity regulator. Adding a proper amount of citric acid to the beverage cannot only improve the taste, but also adjust the color accordingly. However, adding too little amount of citric acid may affect the color and taste of beverage, while adding too much may affect the quality, owing to protein denaturation. The proportion of citric acid is selected at $0.06 \%, 0.08 \%, 0.10 \%, 0.12 \%$, and $0.14 \%$ in our experiment. The effect of citric acid volume on the quality of rose milk beverage is shown in Table 5 .

Table 5 Effect of citric acid amount on the quality of rose milk beverage

\begin{tabular}{ll}
\hline Dosage $(\%)$ & Sensory score/points \\
$0.06 \%$ & 17 \\
$0.08 \%$ & 20 \\
$0.10 \%$ & 24 \\
$0.12 \%$ & 22 \\
$0.14 \%$ & 21 \\
\hline
\end{tabular}

Table 5 shows that when the proportion of citric acid is between $0.06 \%$ and $0.10 \%$ of the total, the sensory evaluation score rises with the increase of citric acid. When the proportion is $10 \%$, the sensory score is the highest. However, when the proportion is between $0.10 \%$ and $0.14 \%$, the score decreases gradually, because it's too sour with darker color.

\subsection{Orthogonal experimental factors and horizontal ratio}

In the experiments, the volume of rose juice, milk, white sugar, and citric acid, the main components of the rose milk beverage, were viewed as experimental factors. Orthogonal experiments were carried out using four factors and three levels, in order to determine the optimal test formula. The test plan is shown in Table 6 .

Table 6 Orthogonal experimental factors of rose milk beverage

\begin{tabular}{ccccc}
\hline \multirow{2}{*}{ Number } & $\mathrm{A}$ & $\mathrm{B}$ & $\mathrm{C}$ & $\mathrm{D}$ \\
\cline { 2 - 5 } & Addition of rose juice (\%) & Milk(\%) & White sugar (\%) & Citric acid (\%) \\
1 & 6 & 4 & 8 & 0.08 \\
2 & 8 & 6 & 10 & 0.10 \\
3 & 10 & 8 & 12 & 0.12 \\
\hline
\end{tabular}


Table 7 Orthogonal experimental results of rose milk beverage

\begin{tabular}{|c|c|c|c|c|c|}
\hline & A & $\mathrm{B}$ & $\mathrm{C}$ & $\mathrm{D}$ & \\
\hline $\mathrm{NO}$ & Addition of rose juice $(\%)$ & $\operatorname{Milk}(\%)$ & White sugar $(\%)$ & Citric acid (\%) & Score \\
\hline 1 & 1（6） & $1(4)$ & 1(8) & $1(0.08)$ & 76 \\
\hline 2 & 1 & $2(6)$ & $2(10)$ & $2(0.10)$ & 84 \\
\hline 3 & 1 & $3(8)$ & $3(12)$ & $3(0.12)$ & 74 \\
\hline 4 & $2(8)$ & 1 & 2 & 3 & 78 \\
\hline 5 & 2 & 2 & 3 & 1 & 79 \\
\hline 6 & 2 & 3 & 1 & 2 & 78 \\
\hline 7 & $3(10)$ & 1 & 3 & 2 & 74 \\
\hline 8 & 3 & 2 & 1 & 3 & 75 \\
\hline 9 & 3 & 3 & 2 & 1 & 74 \\
\hline $\mathrm{K}_{1}$ & 234 & 228 & 229 & 229 & \\
\hline $\mathrm{K}_{2}$ & 235 & 238 & 236 & 236 & \\
\hline $\mathrm{K}_{3}$ & 223 & 226 & 227 & 227 & \\
\hline $\mathrm{K} 1$ & 78 & 76 & 76.3 & 76.3 & \\
\hline K2 & 78.3 & 79.3 & 78.7 & 78.7 & \\
\hline $\mathrm{k} 3$ & 74.3 & 75.3 & 75.7 & 75.7 & \\
\hline \multirow[t]{2}{*}{$\mathrm{R}$} & 4 & 4 & 3 & 3 & \\
\hline & Optimal condition & $\mathrm{A} 2$ & B2 & D2 & \\
\hline
\end{tabular}

Table 7 shows that A2, B2, C2 and D2 is the best combination, that is, the proportions of rose juice, milk, sugar and citric acid are $8 \%, 6 \%, 10 \%$ and $0.1 \%$, respectively.

\section{Conclusion}

First, the rose milk beverage is pink or light pink, sweet and sour, delicate in taste, with the original fragrance of roses, uniform structure and without delamination, sediment and impurities.

Second, the rose milk beverage is processed using a composite stabilizer of $0.15 \%$ sodium alginate and $0.15 \%$ sodium carboxymethylcellulose. The ration of two compound stabilizers is $1: 1$, which can achieve more stable results.

Third, the best experimental formula for the rose milk drink is $8 \%$ rose juice, $6 \%$ milk, $10 \%$ white sugar, and a ratio of 1:1 of compound stabilizer. The test results show that the rose milk beverage processed by this formula has the best quality, color, aroma and texture.

\section{Acknowledgement}

Supported by Science and Technology Plan Project of Yunnan Science and Technology Department (Grant No: 2018FD101)

\section{References}

1. Qiang Sheng.Botany [M].Beijing Higher Education Press, 2006, (3) : 308 309.

2. Zhang Shujun.hennong.Baicao Prescription[M] . Traditional Chinese Medicine Classics Press, 1996, (6) : 61 62.
3. Wan Dequan. Medicinal Botany[M]. Sciences Editions, 2001, (4) : 127 130.

4. Zhao Yali.Study on Processing Technology of Mung Bean Milk Beverage[J]. Beverage Industry, 2012, 15 (8) : 4 15.

5. Wang Li.Food Nutrition[M] .Chemical Industry Press, 2008, (8) : 45 47. 